\title{
Continuous-Wave Single-Photon Transistor Based on a Superconducting Circuit
}

\author{
Oleksandr Kyriienko and Anders S. Sørensen \\ The Niels Bohr Institute, University of Copenhagen, Blegdamsvej 17, DK-2100 Copenhagen, Denmark \\ (Received 21 March 2016; revised manuscript received 8 September 2016; published 28 September 2016)
}

\begin{abstract}
We propose a microwave frequency single-photon transistor which can operate under continuous wave probing and represents an efficient single microwave photon detector. It can be realized using an impedance matched system of a three level artificial ladder-type atom coupled to two microwave cavities connected to input-output waveguides. Using a classical drive on the upper transition, we find parameter space where a single photon control pulse incident on one of the cavities can be fully absorbed into hybridized excited states. This subsequently leads to series of quantum jumps in the upper manifold and the appearance of a photon flux leaving the second cavity through a separate input-output port. The proposal does not require time variation of the probe signals, thus corresponding to a passive version of a single-photon transistor. The resulting device is robust to qubit dephasing processes, possesses low dark count rate for large anharmonicity, and can be readily implemented using current technology.
\end{abstract}

DOI: 10.1103/PhysRevLett.117.140503

Electronic transistors-devices where a weak electrical signal controls a strong probe from a source-lie at the heart of modern electronics, and has led to vast development of classical computing devices. By analogy, in the realm of quantum computing a similar device was contrived, where a single photon control pulse triggers the transmission of a strong coherent probe, and was named a single-photon transistor (SPT) [1]. The operation of the SPT device proposed in Ref. [1] is based on a time-dependent control of the drive and a strong atom-photon interaction. In the optical domain single-photon transistors along these lines were realized with neutral atoms embedded in an optical cavity [2-4], a quantum dot in a waveguide $[5,6]$, or an ultracold gas with Rydberg interactions $[7,8]$. So far the achieved efficiencies have been limited, but if this is improved, a single-photon transistor could represent a powerful tool for coherent state manipulation and quantum information processing [9]. Importantly, a SPT can also serve as an efficient single-photon detector (SPD), as it amplifies a single photon signal by a large gain.

Recently, a microwave frequency range counterpart of quantum optics - circuit quantum electrodynamics (cQED) [10] — has emerged as a highly promising platform for quantum computation [11-14]. Based on high-quality superconducting microwave cavities combined with Josephson junction-based artificial atoms, it enables a strong light-matter coupling even at the single photon level, and allows studying numerous nonlinear microwave quantum optics phenomena [15-18]. The development of a simple and efficient single microwave photon detector is still an open question [19]. The suggested realizations include SPDs based on current-biased Josephson junctions [20], catching an inverted time-controlled pulse [21,22], transmon chain linked with nonreciprocal elements [23], and double quantum dot structures [24]. Also, several schemes for cQED-based single-photon transistors have been proposed $[25,26]$. Ultimately, however, these proposals rely on active time control of the system and input single photon pulse, which complicates the detection process and limits the applicability. Lately such a time dependent protocol based on impedance-matched artificial $\Lambda$ atoms was proposed [27] and experimentally realized [28]. This protocol can be extended to perform time-independent detection [29] if highly anharmonic systems with long coherence time can be constructed.

Here, we propose a single-photon transistor which can operate under continuous wave $(\mathrm{cw})$ probe conditions, where a single photon control pulse triggers an avalanche of gain photons. It represents a passive device which does not require signal and probe timing, largely extending its applicability. The proposed device is robust to imperfections and is particularly insensitive to dephasing. The generic idea relies on a three level ladder atom [Fig. 1(b)], with its lower transition weakly coupled to the first input cavity, and the upper transition strongly driven by a classical source as well as strongly coupled to a second output cavity. A single photon entering the input port transfers the atom to the excited subspace through an impedance matching mechanism similar to Refs. [27-32]. In the excited subspace, a number of quantum jumps between dressed atom-cavity states leads to an enhanced output signal.

System and Hamiltonian.-As a particular realization of cw microwave SPT we propose a superconducting artificial atom with three states $|g\rangle,|e\rangle$, and $|f\rangle$ (qutrit), which is coupled to two separate microwave cavities (modes $\hat{a}_{1}$ and $\hat{a}_{2}$ ), both connected to input-output waveguide channels with coupling constants $\kappa_{1}$ and $\kappa_{2}$ [see sketch in Fig. 1(a)]. The qutrits should have versatile connectivity $[13,33,34]$ and sizeable anharmonicity, making flux [35] and 
(a)

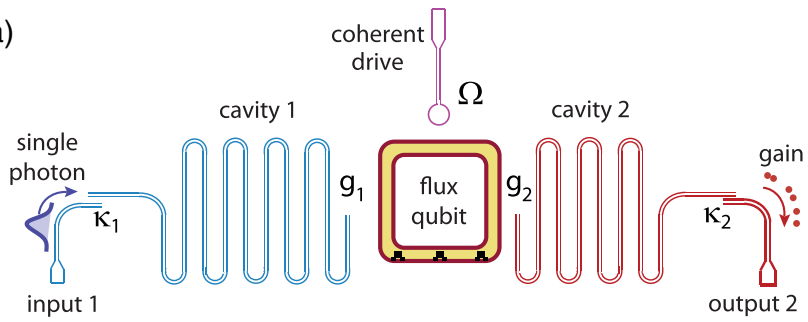

(b)

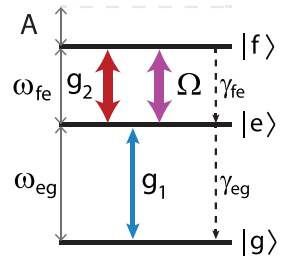

(c)

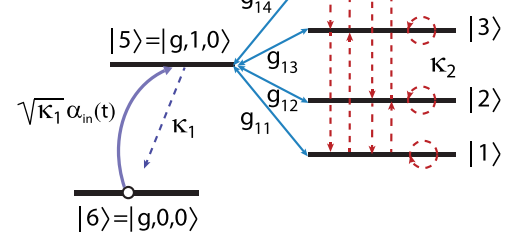

FIG. 1. (a) Sketch of the system, showing a driven qutrit coupled to two superconducting cavities, each connected to a separate input-output line. (b) Energy diagram of three lowest artificial atom levels with associated cavity and drive couplings. (c) Relevant manifold of SPT operational levels with dressed excited states.

fluxonium [36] types desirable. Its lower $|g\rangle-|e\rangle$ transition is resonantly coupled to a cavity mode $\hat{a}_{1}$ with a perturbative coupling $g_{1}$, while the upper $|e\rangle-|f\rangle$ transition is strongly coupled to cavity $\hat{a}_{2}$ with strength $g_{2}$ [Fig. 1(b)]. Additionally, the upper levels are driven by a classical drive of strength $\Omega$.

The Hamiltonian of the system reads

$$
\hat{\mathcal{H}}=\hat{\mathcal{H}}_{\text {sys }}+\hat{\mathcal{H}}_{\mathrm{wgd}, 1}+\hat{\mathcal{H}}_{\mathrm{wgd}, 2},
$$

where $\hat{\mathcal{H}}_{\text {sys }}$ corresponds to the system Hamiltonian written in the rotating frame as [37a]

$$
\begin{aligned}
\hat{\mathcal{H}}_{\mathrm{sys}}= & \delta_{e} \sigma_{\mathrm{ee}}+\left(\delta_{e}+\delta_{f}\right) \sigma_{\mathrm{ff}}+\delta_{\mathrm{cav}, 1} \hat{a}_{1}^{\dagger} \hat{a}_{1}+\delta_{\mathrm{cav}, 2} \hat{a}_{2}^{\dagger} \hat{a}_{2} \\
& +g_{1}\left(\hat{a}_{1}^{\dagger} \sigma_{\mathrm{eg}}^{-}+\sigma_{\mathrm{eg}}^{+} \hat{a}_{1}\right)+g_{2}\left(\hat{a}_{2}^{\dagger} \sigma_{\mathrm{fe}}^{-}+\sigma_{\mathrm{fe}}^{+} \hat{a}_{2}\right) \\
& +\Omega\left(\sigma_{\mathrm{fe}}^{-}+\sigma_{\mathrm{fe}}^{+}\right),
\end{aligned}
$$

with detunings $\delta_{e}=\omega_{\mathrm{eg}}-\omega_{s}, \quad \delta_{f}=\omega_{\mathrm{fe}}-\omega_{d}, \quad \delta_{\mathrm{cav}, 1}=$ $\omega_{\mathrm{cav}, 1}-\omega_{s}$, and $\delta_{\mathrm{cav}, 2}=\omega_{\mathrm{cav}, 2}-\omega_{d}$. Here $\omega_{\mathrm{eg}}, \omega_{\mathrm{fe}}$, $\omega_{\text {cav }, 1}, \omega_{\text {cav }, 2}$ denote energy separations between qutrit levels and energies of microwave cavities, sequentially $(\hbar=1) . \omega_{s}$ is the single input photon central frequency and $\omega_{d}$ is the frequency of the classical drive. $\sigma_{\mathrm{mn}}^{+}=|m\rangle\langle n|$ $\left(\sigma_{\mathrm{mn}}^{-}=|n\rangle\langle m|\right)$ denotes qutrit raising (lowering) operator, and $\sigma_{\mathrm{mm}}=|m\rangle\langle m| . \hat{\mathcal{H}}_{\mathrm{wgd}, \mathrm{j}}$ describes the coupling to the waveguides $j=1,2$ [37a]. The expression for $\hat{\mathcal{H}}_{\text {sys }}$ assumes infinitely large anharmonicity, which precludes parasitic couplings to other-than-resonant qutrit transitions, and we assume long decay and dephasing times for the qutrit. These assumptions will be revisited later. Also, in the following we consider zero detuning for the incoming single photon pulse, $\delta_{e}=\delta_{\mathrm{cav}, 1}=0$, a resonant microwave drive, $\delta_{f}=0$, and a cavity resonant to the upper transition, $\delta_{\text {cav }, 2}=0$.

Operational principle.-First, a single photon pulse enters through the input channel, which is loaded by the joint qubit-cavity system with Hilbert space $\{|g\rangle,|e\rangle,|f\rangle\} \otimes\left|n_{1}\right\rangle \otimes\left|n_{2}\right\rangle \equiv\left|m, n_{1}, n_{2}\right\rangle(m=g, e, f)$. In the input stage we consider a weak excitation such that $n_{1}$ is restricted to vacuum or a single excitation. Then, the relevant subspace of states contains the ground state $|g, 0,0\rangle$, the first cavity excited state $|g, 1,0\rangle$, and the subspace $\left\{\left|e, 0, n_{2}\right\rangle,\left|f, 0, n_{2}\right\rangle\right\}$ which we call excited states. Because of the strong couplings $g_{2}$ and $\Omega$, the excited states become hybridized, and it is convenient to introduce dressed metastable states. For the lowest cavity 2 occupation with $n_{2}=0,1$ this embeds a subspace $\mathcal{M}=$ $\{|1\rangle,|2\rangle,|3\rangle,|4\rangle\}$ (Fig. 1(c) and [37b]), and higher states can be included analogously. Because of the admixture of the $|e, 0,0\rangle$ level, each dressed state is coupled to $|g, 1,0\rangle$ with a modified constant $g_{1 m}$. Considering $g_{1}$ to be perturbative, the coupling of the first cavity to the dressed states $\mathcal{M}$ works as an effective decay channel. By controlling the drive strength $\Omega$ and coupling parameters, this effective total decay rate $\Gamma_{\text {set }}$ can be made equal to the coupling of the cavity to the first waveguide $\kappa_{1}$, reducing the system to an impedance matched $\Lambda$ system attached to a single-sided waveguide [37c]. Such impedance matching (IM) has already proven to be useful for cQED circuits, leading to photo detection [28], as well as proposals for microwave downconversion [30,31] and gates for flying qubits [32]. This allows for a full absorption of a single photon pulse by the metastable states, leading to near-unity single photon switching and photon detection without the need for temporally varying control fields.

Once the setting stage to the excited states manifold is completed, the second decay channel $\kappa_{2}$ leads to quantum jumps between the dressed states [Fig. 1(a), red dashed lines], where a series of jumps within the full $\left\{\left|e, 0, n_{2}\right\rangle,\left|f, 0, n_{2}\right\rangle\right\}$ $\left(n_{2}=0,1, \ldots, \mathcal{N}_{2}\right)$ subspace occur. Radiative jumps between the states lead to a flux leaving cavity 2 through the output port, resulting in an enhancement of the signal. Once the system recovers to the ground state $|g, 0,0\rangle$, the SPT duty cycle is finalized. The SPT can thus serve as a highly efficient single-photon detector, and corresponds to a microwave version of a single photon avalanche diode.

Input: impedance matching.-First, we characterize the single photon input stage. To define the IM condition analytically we exploit the effective operator theory [38] to estimate the decay rate $\Gamma_{\text {set }}$ from $|g, 1,0\rangle$ to the metastable states [37d]. Considering $g_{1}<g_{2}, \Omega$ and up to $\mathcal{N}_{2}=2$ photons, we find

$$
\Gamma_{\text {set }}=\frac{16 \kappa_{2} g_{1}^{2} g_{2}^{2}\left(16 g_{2}^{2}+4 \kappa_{2}^{2}+\Omega^{2}\right)}{4 \kappa_{2}^{2}\left(4 g_{2}^{2}+\kappa_{2}^{2}\right) \Omega^{2}+5 \kappa_{2}^{2} \Omega^{4}+\Omega^{6}},
$$



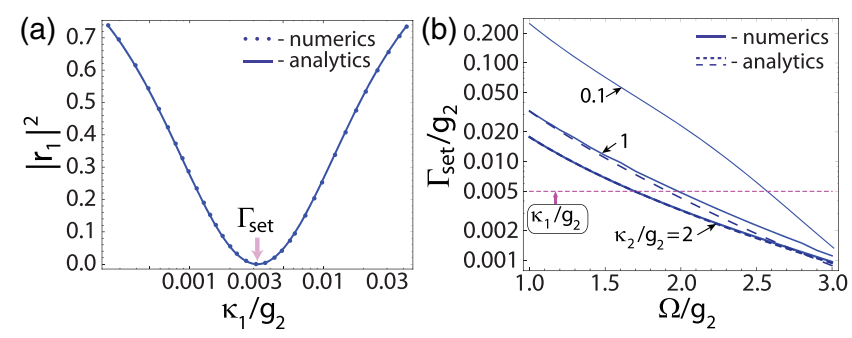

FIG. 2. (a) Reflection coefficient for the first cavity as a function of input coupling rate $\kappa_{1}$ (log scale). The dotted line is a full numerical calculation, and the solid line represents Eq. (4) with $\Gamma_{\text {set }}$ given by Eq. (3). Here $\Omega / g_{2}=2, g_{1} / g_{2}=0.05$, and $\kappa_{2} / g_{2}=2$. (b) Tunability of the effective setting rate as a function of drive strength $\Omega$ and varying $\kappa_{2}$ with $g_{1} / g_{2}=0.05$. The full lines are the result of a numerical simulation which agree with the analytical prediction of Eq. (4) for $\kappa_{2} \gtrsim 1$. The dashed horizontal line represents the impedance matching condition which can always be obtained by varying $\Omega$.

which provides a good estimate for $\kappa_{2} / g_{2} \gtrsim 1.5$, where the relevant processes happen within the lowest Fock states. The setting rate for smaller $\kappa_{2} / g_{2}$ can be derived by increasing $\mathcal{N}_{2}$ [37d].

To test the IM condition numerically, we exploit the input-output theory for the Hamiltonian (1) by deriving the Heisenberg equations of motion for the system operators, and assuming a weak coherent input. The efficiency of the setting stage is quantified by numerically calculating the reflection coefficient $\left|r_{1}\right|^{2}=\left|\left\langle\hat{a}_{\text {out }, 1}\right\rangle /\left\langle\hat{a}_{\text {in, } 1}\right\rangle\right|^{2}$ at the input port. In the simulation a reflection minimum is obtained at the impedance matched setting rate $\kappa_{1}=\Gamma_{\text {set }}$ [Fig. 2(a)]. The dotted curve corresponds to the full Heisenberg equation calculation, and the solid curve shows the analytical solution for a reflection coefficient of a waveguide coupled to a $\Lambda$ system [37c,39],

$$
\left|r_{1}\right|^{2}=\frac{\left(\Gamma_{\mathrm{set}} / \kappa_{1}-1\right)^{2}}{\left(\Gamma_{\mathrm{set}} / \kappa_{1}+1\right)^{2}},
$$

with $\Gamma_{\text {set }}$ provided by Eq. (3). The dependence of the setting rate on the classical microwave drive strength $\Omega$ allows fine tuning the IM condition. In Fig. 2(b) we show the dependence of $\Gamma_{\text {set }} / g_{2}$ on $\Omega$, fixing $\kappa_{1}=0.005 g_{2}$ and $g_{1}=0.05 g_{2}$. Plotting the setting rate for three different values of $\kappa_{2}$ we can find a value of $\Omega / g_{2}$ for which IM holds.

Output: Gain of SPT.-To describe the gain of the transistor, we exploit the Heisenberg equations of motion derived using the input-output relations for two coupling channels combined with a Gaussian-shaped single photon pulse [37e]. For temporal widths of the pulse being larger than $\tau>\kappa_{1}^{-1}$ and IM arranged, an incoming single photon excitation is fully transferred to the excited states of the qutrit which are highly mixed with the mode of cavity 2 . Averaging the Heisenberg equation with the wave function
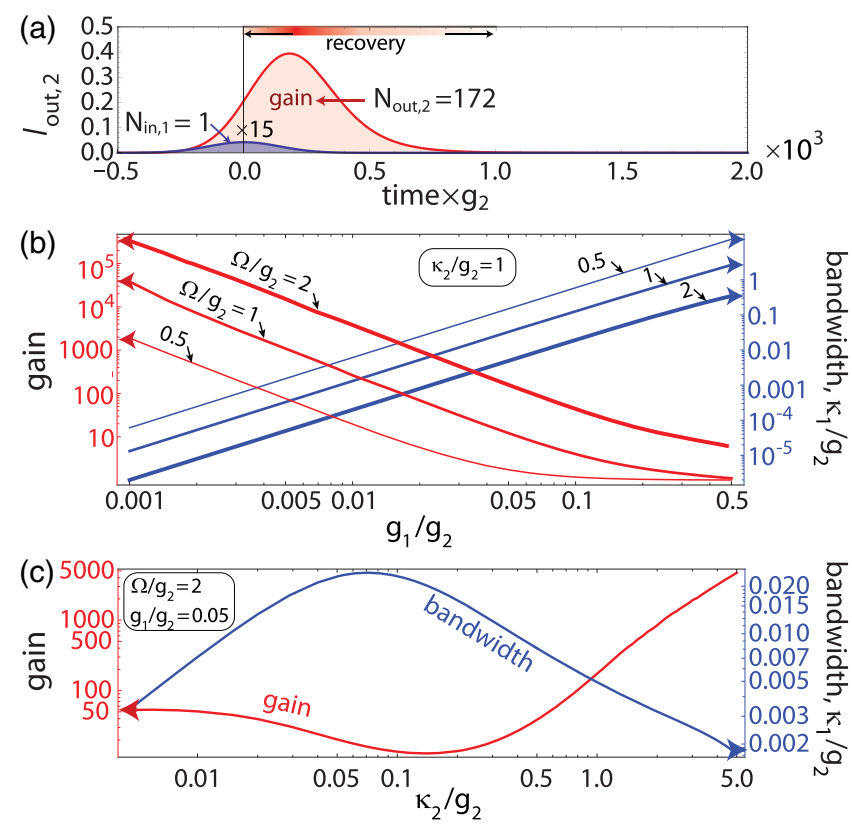

FIG. 3. (a) Time dependence of second cavity output calculated for Gaussian single photon pulse incident on the first cavity; $g_{1} / g_{2}=0.05, \Omega / g_{2}=2, \kappa_{2} / g_{2}=1$. (b) SPT gain and bandwidth plotted as function of the first cavity coupling $g_{1}$ for various drive strengths; $\kappa_{1}=\Gamma_{\text {set }}, \kappa_{2} / g_{2}=1$. (c) SPT gain and bandwidth for varying output coupling $\kappa_{2} ; g_{1} / g_{2}=0.05$.

corresponding to the single photon input $\left|\Psi_{\text {in }}\right\rangle$, we can extract the intensity of outgoing photons in the second waveguide, $\left\langle\hat{a}_{\text {out }, 2}^{\dagger} \hat{a}_{\text {out }, 2}\right\rangle=\kappa_{2}\left\langle\hat{a}_{2}^{\dagger} \hat{a}_{2}\right\rangle \equiv I_{\text {out }, 2}$. In Fig. 3(a) we plot the temporal dependence of input 1 and output 2 photon numbers. For a long Gaussian pulse containing a single photon $\left[N_{\mathrm{in}, 1}=\int d t I_{\mathrm{in}, 1}(t)=1\right]$, we can on average get $N_{\text {out }, 2}=\int d t I_{\text {out }, 2}$ photons at the output of the second cavity before the system recovers to the ground state. This represents the gain of the single-photon transistor, which describes the effective amplification of the single photon signal. The full counting statistics can be obtained by changing to the wave-function Monte Carlo approach. This confirms that a single input photon leads to numerous emitted photons [37f].

To investigate the gain we consider the input stage to be completed, setting $\left|\Psi_{\text {start }}\right\rangle=|e, 0,0\rangle$, and calculate the occupation of cavity 2 using a density matrix approach, truncating the cavity Fock space at $\mathcal{N}_{2}=10$ excitations. The gain is highly sensitive to the system parameters: cavity 1 to qutrit coupling $g_{1}$, microwave drive strength $\Omega$, and output coupling $\kappa_{2}$. Additionally, these parameters set the optimal (IM) value for the input coupling $\kappa_{1}$, and thereby the bandwidth of the single-photon detector. In Fig. 3(b) we show the gain and bandwidth as a function of the coupling $g_{1}$ for varying $\Omega / g_{2}$. The plot shows a fast increase of the bandwidth with the coupling constant $g_{1}$, as it sets the rate at which single photons can get to the excited subspace. Reciprocally, this corresponds to a growth of the 

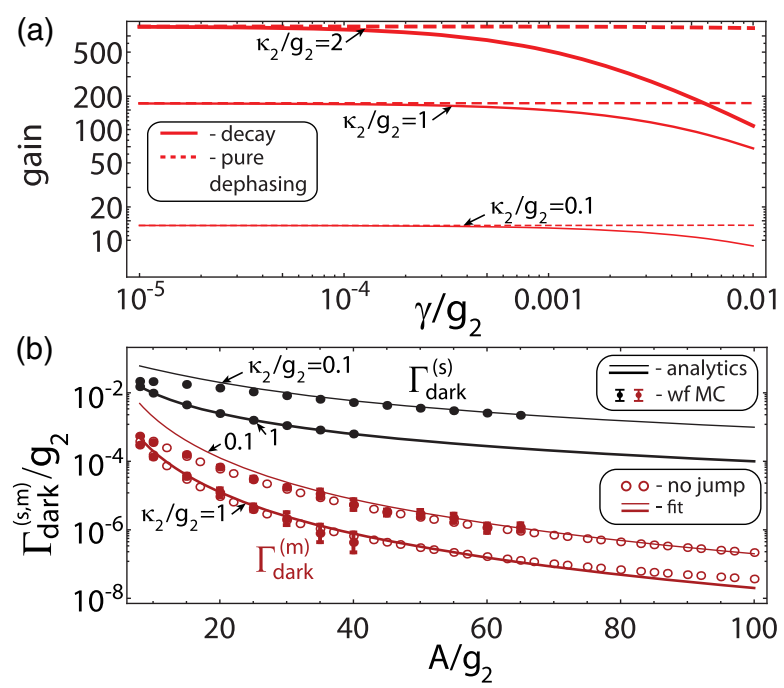

FIG. 4. (a) Gain of SPT as a function of decay (solid curves) and pure dephasing rates (dashed lines); $\Omega / g_{2}=2, g_{1} / g_{2}=0.05$, $\kappa_{1}=\Gamma_{\text {set. }}$. (b) The SPT enhanced $\Gamma_{\text {dark }}^{(\mathrm{m})}$ (lower two curves) and single $\Gamma_{\text {dark }}^{(\mathrm{s})}$ (upper two curves) dark count rates as a function of the qutrit anharmonicity $A$. Here, $\Omega / g_{2}=2, g_{1} / g_{2}=0.05$. Numerical results were obtained using the wave function Monte Carlo approach and studying no-jump evolution of the system [37h]. We show analytical results for a single dark count rate derived using effective operator formalism [Eq. (6), black solid curves]. For $\Gamma_{\text {dark }}^{(\mathrm{m})}$ we also show a fit $\eta \Gamma_{\text {dark,ss }}^{(\mathrm{m})}$ with $\eta \approx 4$.

recovery rate and a reduction of the gain. Thus, there is a trade-off between gain and bandwidth, indicating that a medium $g_{1} / g_{2}$ ratio is favored for optimal detection.

The gain and bandwidth dependence as a function of $\kappa_{2}$ is shown in Fig. 3(c). Choosing $g_{1} / g_{2}=0.05$ and $\Omega / g_{2}=2$ in order to get high input bandwidth, we find that both gain and bandwidth are nonmonotonic functions of $\kappa_{2}$. In particular, we are interested in the region of $0.5<\kappa_{2} / g_{2}<3$, where the gain increases rapidly, while $\kappa_{1}$ is relatively large.

Imperfections.-We now turn to imperfections of the scheme. First, we plot the SPT gain as a function of qubit decay (solid line) and pure dephasing (dashed line) rate $\gamma$ [37g] for three values of $\kappa_{2} / g_{2}$ [see Fig. 4(a)]. While strong decay naturally tends to decrease the gain by introducing an extra nonradiative recovery channel, pure dephasing does not influence the gain, thus removing the need for long qubit coherence time, as opposed to e.g. Refs. [26,27]. We also find that even for larger qubit decay the IM conditions can be always satisfied.

Second, we account for a finite qutrit anharmonicity $A$ and introduce the residual qutrit-cavity couplings defined by the Hamiltonian [37h]

$$
\hat{\mathcal{H}}_{\mathrm{res}}=\left(\sqrt{2} g_{1} \hat{a}_{1}^{\dagger} \sigma_{\mathrm{fe}}^{-}+\frac{g_{2}}{\sqrt{2}} \hat{a}_{2}^{\dagger} \sigma_{\mathrm{eg}}^{-}+\frac{\Omega}{2 \sqrt{2}} \sigma_{\mathrm{fe}}^{-}+\text {H.c. }\right),
$$

and the associated detunings defined by the rotated energy frame of $\hat{\mathcal{H}}_{\mathrm{A}}=A \sigma_{\mathrm{ee}}+A \sigma_{\mathrm{ff}}+A \hat{a}_{1}^{\dagger} \hat{a}_{1}$. Here, the extra terms which couple the ground and the excited states in the absence of a signal photon lead to a nonzero dark count rate of the detector.

There are two separate dark count rates induced by the driving of the lower transition in the system as contained in Eq. (2). The first involves a cycle $|g, 0,0\rangle-|e, 0,0\rangle-$ $|g, 0,1\rangle-|g, 0,0\rangle$ with emission of a single photon at a rate $\Gamma_{\text {dark }}^{(\mathrm{s})}$. Importantly, this process does not include radiative transitions within the excited manifold. Thus it is not amplified by SPT, and can be discriminated for large SPT gains. The second process corresponds to ground-to-excited state transitions $|g, 0,0\rangle-|e, 0,0\rangle-|g, 0,1\rangle-|e, 0,1\rangle-$ $|e, 0,0\rangle$ happening at a rate $\Gamma_{\text {dark }}^{(\mathrm{m})}$, which projects the system to the excited manifold, launches a photon avalanche, and needs to be strongly suppressed.

To access the rates directly we find the full counting statistics using the wave function Monte Carlo approach and study the no-jump evolution of the system [37h], allowing us to estimate the dark count rates for the single and enhanced processes. The results are shown in Fig. 4(b). Additionally, the steady state dark count rates can be calculated using an effective operator approach [37h], valid for large anharmonicities, which gives

$$
\begin{gathered}
\Gamma_{\text {dark,ss }}^{(\mathrm{s})} \approx \frac{\kappa_{2} g_{2}^{2} \Omega^{2}}{4\left(A^{2} \kappa_{2}^{2}+g_{2}^{4}\right)}, \\
\Gamma_{\text {dark,ss }}^{(\mathrm{m})} \approx \frac{g_{2}^{2} \Omega^{4}}{32 A^{4} \kappa_{2}} .
\end{gathered}
$$

While the simplified analytical result for $\Gamma_{\text {dark,ss }}^{(\mathrm{s})}$ coincides with numerical estimates for $A / g_{2}>40$, the enhanced dark count rate $\Gamma_{\text {dark }}^{(\mathrm{m})}$ shows an additional dynamical contribution due to induced jumps to the excited subspace triggered by the jumps with the rate $\Gamma_{\text {dark,ss }}^{(\mathrm{s})}$. From the numerical simulation we find that the total rate $\Gamma_{\text {dark }}^{(\mathrm{m})}$ retains the favorable $A^{-4}$ scaling but is roughly a factor of $\eta \approx 4$ larger than $\Gamma_{\text {dark,ss }}^{(\mathrm{m})}[37 \mathrm{~h}]$.

Real structure estimates.-For a realistic example we consider a flux qutrit where $g_{2}=2 \pi \times 458 \mathrm{MHz}$ can be attained for an anharmonicity $A=2 \pi \times 8.426 \mathrm{GHz}$ and a decay rate $\gamma=2 \pi \times 0.227 \mathrm{MHz}$ [30]. Decreasing $g_{2}$ to $2 \pi \times 120 \mathrm{MHz}$, setting $\kappa_{2}=g_{2}, g_{1}=2 \pi \times 6 \mathrm{MHz}$, and $\Omega=2 \pi \times 240 \mathrm{MHz}$ gives a gain of 172 photons with $2 \pi \times 0.6 \mathrm{MHz}$ bandwidth, enhanced dark count rate of $2 \pi \times 660 \mathrm{~Hz}$, and single dark count rate $2 \pi \times 14.4 \mathrm{kHz}$.

Finally, we note that for using the device as a singlephoton detector the gain photons need to be measured. For the above scenario with a gain of approximately 200 we estimate that the signal is distributed on $\sim 90$ modes. With a heterodyne detection setup this output field can be 
measured with an efficiency of $95 \%$ with only a 0.02 dark count probability and better performance can be achieved at higher gain [37i]. Alternatively the expected signal begins to be within range of calorimetric detection schemes [40].

Conclusion.-We have presented a scheme for a singlephoton transistor based on the impedance-matched superconducting circuit, which operates in the cw regime and allows for an on-demand single microwave photon detection. The scheme can realistically lead to an output of several hundred photons, tolerates high pure dephasing rates, and keeps low dark count rates.

The research was funded by the European Union Seventh Framework Programme through the ERC Grant QIOS (Grant No. 306576).

[1] D. E. Chang, A. S. Sørensen, E. A. Demler, and M. D. Lukin, A single-photon transistor using nanoscale surface plasmons, Nat. Phys. 3, 807 (2007).

[2] W. Chen, K. M. Beck, R. Bücker, M. Gullans, M. D. Lukin, H. Tanji-Suzuki, and V. Vuletić, All-optical switch and transistor gated by one stored photon, Science 341, 768 (2013).

[3] T. G. Tiecke, J. D. Thompson, N. P. de Leon, L. R. Liu, V. Vuletić, and M. D. Lukin, Nanophotonic quantum phase switch with a single atom, Nature (London) 508, 241 (2014).

[4] A. Reiserer, N. Kalb, G. Rempe, and S. Ritter, A quantum gate between a flying optical photon and a single trapped atom, Nature (London) 508, 237 (2014).

[5] H. Kim, R. Bose, T. C. Shen, G. S. Solomon, and E. Waks, A quantum logic gate between a solid-state quantum bit and a photon, Nat. Photonics 7, 373 (2013).

[6] A. Javadi, I. Söllner, M. Arcari, S. Lindskov Hansen, L. Midolo, S. Mahmoodian, G. Kiršanske, T. Pregnolato, E. H. Lee, J. D. Song, S. Stobbe, and P. Lodahl, Single-photon nonlinear optics with a quantum dot in a waveguide, Nat. Commun. 6, 8655 (2015).

[7] D. Tiarks, S. Baur, K. Schneider, S. Dürr, and G. Rempe, Single-Photon Transistor Using a Förster Resonance, Phys. Rev. Lett. 113, 053602 (2014).

[8] H. Gorniaczyk, C. Tresp, J. Schmidt, H. Fedder, and S. Hofferberth, Single-Photon Transistor Mediated by Interstate Rydberg Interactions, Phys. Rev. Lett. 113, 053601 (2014).

[9] L.-M. Duan and H. J. Kimble, Scalable Photonic Quantum Computation through Cavity-Assisted Interactions, Phys. Rev. Lett. 92, 127902 (2004).

[10] A. Wallraff, D. I. Schuster, A. Blais, L. Frunzio, R.-S. Huang, J. Majer, S. Kumar, S. M. Girvin, and R. J. Schoelkopf, Circuit quantum electrodynamics: Coherent coupling of a single photon to a Cooper pair box, Nature (London) 431, 162 (2004).

[11] R. Barends, J. Kelly, A. Megrant, A. Veitia, D. Sank, E. Jeffrey, T. C. White, J. Mutus, A. G. Fowler, B. Campbell, Y. Chen, Z. Chen, B. Chiaro, A. Dunsworth, C. Neill, P. O’Malley, P. Roushan, A. Vainsencher, J. Wenner, A. N. Korotkov, A. N. Cleland, and J.M. Martinis, Superconducting quantum circuits at the surface code threshold for fault tolerance, Nature (London) 508, 500 (2014).

[12] J. Kelly, R. Barends, A. G. Fowler, A. Megrant, E. Jeffrey, T. C. White, D. Sank, J. Y. Mutus, B. Campbell, Yu Chen, Z. Chen, B. Chiaro, A. Dunsworth, I.-C. Hoi, C. Neill, P. J. J. O'Malley, C. Quintana, P. Roushan, A. Vainsencher, J. Wenner, A. N. Cleland, and John M. Martinis, State preservation by repetitive error detection in a superconducting quantum circuit, Nature (London) 519, 66 (2015).

[13] D. Risté, S. Poletto, M.-Z. Huang, A. Bruno, V. Vesterinen, O.-P. Saira, and L. DiCarlo, Detecting bit-flip errors in a logical qubit using stabilizer measurements, Nat. Commun. 6, 6983 (2015).

[14] M. D. Reed, L. DiCarlo, S. Nigg, L. Sun, L. Frunzio, S. M. Girvin, and R. J. Schoelkopf, Realization of three-qubit quantum error correction with superconducting circuits, Nature (London) 482, 382 (2012).

[15] Lev S. Bishop, J. M. Chow, J. Koch, A. A. Houck, M. H. Devoret, E. Thuneberg, S. M. Girvin, and R. J. Schoelkopf, Nonlinear response of the vacuum Rabi resonance, Nat. Phys. 5, 105 (2009).

[16] A. A. Houck, D. I. Schuster, J. M. Gambetta, J. A. Schreier, B. R. Johnson, J. M. Chow, L. Frunzio, J. Majer, M. H. Devoret, S. M. Girvin, and R. J. Schoelkopf, Generating single microwave photons in a circuit, Nature (London) 449, 328 (2007).

[17] Io-Chun Hoi, A. F. Kockum, T. Palomaki, T. M. Stace, B. Fan, L. Tornberg, S. R. Sathyamoorthy, G. Johansson, P. Delsing, and C.M. Wilson, Giant CrossKerr Effect for Propagating Microwaves Induced by an Artificial Atom, Phys. Rev. Lett. 111, 053601 (2013).

[18] M. Pechal, L. Huthmacher, C. Eichler, S. Zeytinoğlu, A. A. Abdumalikov, Jr., S. Berger, A. Wallraff, and S. Filipp, Microwave-Controlled Generation of Shaped Single Photons in Circuit Quantum Electrodynamics, Phys. Rev. X 4, 041010 (2014).

[19] S. R. Sathyamoorthy, T. M. Stace, and G. Johansson, Detecting itinerant single microwave photons, arXiv:1504 .04979

[20] G. Romero, J. J. García-Ripoll, and E. Solano, Microwave Photon Detector in Circuit QED, Phys. Rev. Lett. 102, 173602 (2009).

[21] Y. Yin, Y. Chen, D. Sank, P. J. J. O’Malley, T. C. White, R. Barends, J. Kelly, E. Lucero, M. Mariantoni, A. Megrant, C. Neill, A. Vainsencher, J. Wenner, Alexander N. Korotkov, A. N. Cleland, and John M. Martinis, Catch and Release of Microwave Photon States, Phys. Rev. Lett. 110, 107001 (2013).

[22] J. Wenner, Yi Yin, Yu Chen, R. Barends, B. Chiaro, E. Jeffrey, J. Kelly, A. Megrant, J. Y. Mutus, C. Neill, P. J. J. O'Malley, P. Roushan, D. Sank, A. Vainsencher, T. C. White, Alexander N. Korotkov, A. N. Cleland, and John M. Martinis, Catching Time-Reversed Microwave Coherent State Photons with 99.4\% Absorption Efficiency, Phys. Rev. Lett. 112, 210501 (2014).

[23] S. R. Sathyamoorthy, L. Tornberg, A. F. Kockum, B. Q. Baragiola, J. Combes, C. M. Wilson, T. M. Stace, and G. Johansson, Quantum Nondemolition Detection of a Propagating Microwave Photon, Phys. Rev. Lett. 112, 093601 (2014). 
[24] C. H. Wong and M. G. Vavilov, Quantum efficiency of a microwave photon detector based on a double quantum dot, arXiv:1512.06939.

[25] L. Neumeier, M. Leib, and M. J. Hartmann, Single-Photon Transistor in Circuit Quantum Electrodynamics, Phys. Rev. Lett. 111, 063601 (2013).

[26] M. T. Manzoni, F. Reiter, J. M. Taylor, and A. S. Sørensen, Single-photon transistor based on superconducting systems, Phys. Rev. B 89, 180502(R) (2014).

[27] K. Koshino, K. Inomata, Z. Lin, Y. Nakamura, and T. Yamamoto, Theory of microwave single-photon detection using an impedance-matched $\Lambda$ system, Phys. Rev. A 91, 043805 (2015).

[28] K. Inomata, Z. Lin, K. Koshino, W. D. Oliver, J.-S. Tsai, T. Yamamoto, and Y. Nakamura, Single microwave-photon detector using an artificial $\Lambda$-type three-level system, Nat. Commun. 7, 12303 (2016).

[29] K. Koshino, Z. Lin, K. Inomata, T. Yamamoto, and Y. Nakamura, Dressed-state engineering for continuous detection of itinerant microwave photons, Phys. Rev. A 93, 023824 (2016).

[30] K. Inomata, K. Koshino, Z. R. Lin, W. D. Oliver, J.-S. Tsai, Y. Nakamura, and T. Yamamoto, Microwave DownConversion with an Impedance-Matched $\Lambda$ System in Driven Circuit QED, Phys. Rev. Lett. 113, 063604 (2014).

[31] K. Koshino, K. Inomata, T. Yamamoto, and Y. Nakamura, Implementation of an Impedance-Matched $\Lambda$ System by Dressed-State Engineering, Phys. Rev. Lett. 111, 153601 (2013).

[32] K. Koshino, S. Ishizaka, and Y. Nakamura, Deterministic photon-photon $\sqrt{\mathrm{SWAP}}$ gate using a $\Lambda$ system, Phys. Rev. A 82, 010301(R) (2010).

[33] R. Barends, J. Kelly, A. Megrant, D. Sank, E. Jeffrey, Y. Chen, Y. Yin, B. Chiaro, J. Mutus, C. Neill, P. O'Malley, P. Roushan, J. Wenner, T. C. White, A. N. Cleland, and J. M. Martinis, Coherent Josephson Qubit Suitable for Scalable Quantum Integrated Circuits, Phys. Rev. Lett. 111, 080502 (2013).

[34] Y. Chen, C. Neill, P. Roushan, N. Leung, M. Fang, R. Barends, J. Kelly, B. Campbell, Z. Chen, B. Chiaro, A. Dunsworth, E. Jeffrey, A. Megrant, J. Y. Mutus, P. J. J. O’Malley, C. M. Quintana, D. Sank, A. Vainsencher, J. Wenner, T. C. White, M. R. Geller, A. N. Cleland, and J. M. Martinis, Qubit Architecture with High Coherence and Fast Tunable Coupling, Phys. Rev. Lett. 113, 220502 (2014).

[35] I. Chiorescu, P. Bertet, K. Semba, Y. Nakamura, C. J. P. M. Harmans, and J. E. Mooij, Coherent dynamics of a flux qubit coupled to a harmonic oscillator, Nature (London) 431, 159 (2004).

[36] V.E. Manucharyan, J. Koch, L. I. Glazman, and M. H. Devoret, Fluxonium: Single Cooper-pair circuit free of charge offsets, Science 326, 113 (2009).

[37] See Supplemental Material at http://link.aps.org/ supplemental/10.1103/PhysRevLett.117.140503 for (a) transformation of original Hamiltonian to the rotating frame; (b) excited states diagonalization; (c) IM $\Lambda$ atom analogy; (d) effective setting rate derivation; (e) Heisenberg equations derivation; (f) wave function Monte Carlo calculations and source statistics; (g) definition of decay and decoherence rates; (h) full dark count rates description; and (i) heterodyne detection scheme. The associated references are given by [38-51].

[38] F. Reiter and A. S. Sørensen, Effective operator formalism for open quantum systems, Phys. Rev. A 85, 032111 (2012).

[39] D. Pinotsi and A. Imamoglu, Single Photon Absorption by a Single Quantum Emitter, Phys. Rev. Lett. 100, 093603 (2008).

[40] J. Govenius, R. E. Lake, K. Y. Tan, and M. Möttönen, Detection of Zeptojoule Microwave Pulses Using Electrothermal Feedback in Proximity-Induced Josephson Junctions, Phys. Rev. Lett. 117, 030802 (2016).

[41] A. A. Clerk, M. H. Devoret, S. M. Girvin, F. Marquardt, and R. J. Schoelkopf, Introduction to quantum noise, measurement, and amplification, Rev. Mod. Phys. 82, 1155 (2010).

[42] D. F. Walls and G. J. Milburn, Quantum Optics, 2nd ed. (Springer, New York, 2008).

[43] S. Fan, Ş. E. Kocabaş, and J.-T. Shen, Input-output formalism for few-photon transport in one-dimensional nanophotonic waveguides coupled to a qubit, Phys. Rev. A 82, 063821 (2010).

[44] D. Witthaut and A. S. Sørensen, Photon scattering by a three-level emitter in a one-dimensional waveguide, New J. Phys. 12, 043052 (2010).

[45] J. Dalibard, Y. Castin, and K. Mølmer, Wave-Function Approach to Dissipative Processes in Quantum Optics, Phys. Rev. Lett. 68, 580 (1992).

[46] K. Mølmer, Y. Castin, and J. Dalibard, Monte Carlo wavefunction method in quantum optics, J. Opt. Soc. Am. B 10, 524 (1993).

[47] L. Davidovich, Sub-Poissonian processes in quantum optics, Rev. Mod. Phys. 68, 127 (1996).

[48] J. Koch, T. M. Yu, J. Gambetta, A. A. Houck, D. I. Schuster, J. Majer, A. Blais, M. H. Devoret, S. M. Girvin, and R. J. Schoelkopf, Charge-insensitive qubit design derived from the Cooper pair box, Phys. Rev. A 76, 042319 (2007).

[49] Z. Y. Ou and H. J. Kimble, Probability distribution of photoelectric currents in photodetection processes and its connection to the measurement of a quantum state, Phys. Rev. A 52, 3126 (1995).

[50] C. Iaconis, E. Mukamel, and I. A. Walmsley, Temporal heterodyne detector for multitemporal mode quantum state measurement, J. Opt. B 2, 510 (2000).

[51] F. Mallet, M. A. Castellanos-Beltran, H. S. Ku, S. Glancy, E. Knill, K. D. Irwin, G. C. Hilton, L. R. Vale, and K. W. Lehnert, Quantum State Tomography of an Itinerant Squeezed Microwave Field, Phys. Rev. Lett. 106, 220502 (2011). 